\title{
OBTENÇÃO DA PERMABILIDADE DE MEIOS POROSOS EM TESTES DE PERCOLAÇÃO EM ALTAS PRESSÕES
}

\author{
A. Q. MAGIONI ${ }^{1}$, H. J. OLIVEIRA ${ }^{1}$, D.B.R. SILVA ${ }^{1}$, F.O. AROUCA ${ }^{1}$ e J.J.R. \\ DAMASCENO $^{1}$
}

${ }^{1}$ Universidade Federal de Uberlândia, Faculdade de Engenharia Química

E-mail:_dricamagioni@hotmail.com, heliojuniorcom@yahoo.com, debora_beatriz@hotmail.com, arouca@feq.ufu.br,damasceno@feq.ufu.br.

RESUMO: O entendimento do processo de percolação do fluido de perfuração em rochas-reservatório durante a prospecção de petróleo e os mecanismos envolvidos na formação do reboco da parede do poço como ação reparadora têm despertado o crescente interesse nos estudos acerca do assunto. No processo de perfuração de poços de petróleo em elevadas pressões ocorre naturalmente uma perda do fluido de perfuração que permeia o interior da formação rochosa da parede do poço, causando perdas significativas de materiais no processo. Uma alternativa eficiente para evitar tais perdas, consiste em promover a formação de uma fina camada de baixa permeabilidade, denominada de reboco, que se forma na parede do poço durante a percolação do fluido de perfuração contendo um agente obturante. O presente trabalho buscou obter a permeabilidade de meios porosos de agentes obturantes, tais como o Carbonato de Cálcio, em testes de percolação em altas pressões usando um filtro do tipo HTHP. Tal filtro consiste em um equipamento comumente utilizado na indústria de petróleo para o estudo da filtração estática. Os resultados se mostraram bastante promissores, indicando diferenças significativas na permeabilidade com aumento da pressão.

\section{INTRODUÇÃO}

A filtração é uma operação unitária presente no processo de perfuração de poços de petróleo, neste sistema o fluido de perfuração é injetado pelo interior da coluna de perfuração e retorna a superfície pelo espaço anular existente entre a coluna de perfuração e a formação rochosa. O retorno do fluido de perfuração ocorre em um movimento ascendente (e, conseqüentemente, na direção axial), transportando o cascalho, lubrificando a broca e garantindo a pressão hidrostática no meio.

Sendo a formação rochosa um meio poroso, há uma tendência do fluido de perfuração permear para o interior dessa formação. Esta invasão de fluido de perfuração na formação rochosa é uma ação indesejada, podendo comprometer a amostragem e, conseqüentemente, a avaliação do óleo (Bourgoyne et al., 1991).

Uma maneira de evitar essa ação indesejada é o fluido de perfuração transportar agentes obturantes (e outros aditivos) até as gargantas de poros da rocha-reservatório e, com isso, em condições apropriadas, estabelecer a obstrução do meio poroso que permite a perda de materiais. A filtração do fluido de perfuração na formação rochosa contendo o agente obturante promove a formação de uma camada fina e de baixa permeabilidade denominada de reboco. 
Os estudos relacionados às aplicações clássicas envolvendo a filtração com formação de torta compressível defrontam-se com a dificuldade experimental no levantamento do perfil de porosidades e da dependência entre a porosidade, pressão nos sólidos e permeabilidade (Massarani, 2002).

Neste contexto, o presente trabalhou buscou em testes de percolação em altas pressões usando um filtro do tipo HTHP, onde o agente obturante é o carbonato de cálcio, obter a permeabilidade de meios porosos.

\section{MATERIAL E MÉTODOS}

\subsection{Material}

A avaliação resistividade média da torta, resistência do meio filtrante, porosidade,tempo de filtração e volume de filtrado, é desejável para a obtenção da permeabilidade da torta.

Neste trabalho os ensaios de separação sólido-líquido são realizados utilizando um filtro prensa de alta temperatura e alta pressão (Ofite Dynamic High-Pressure - HTHP).

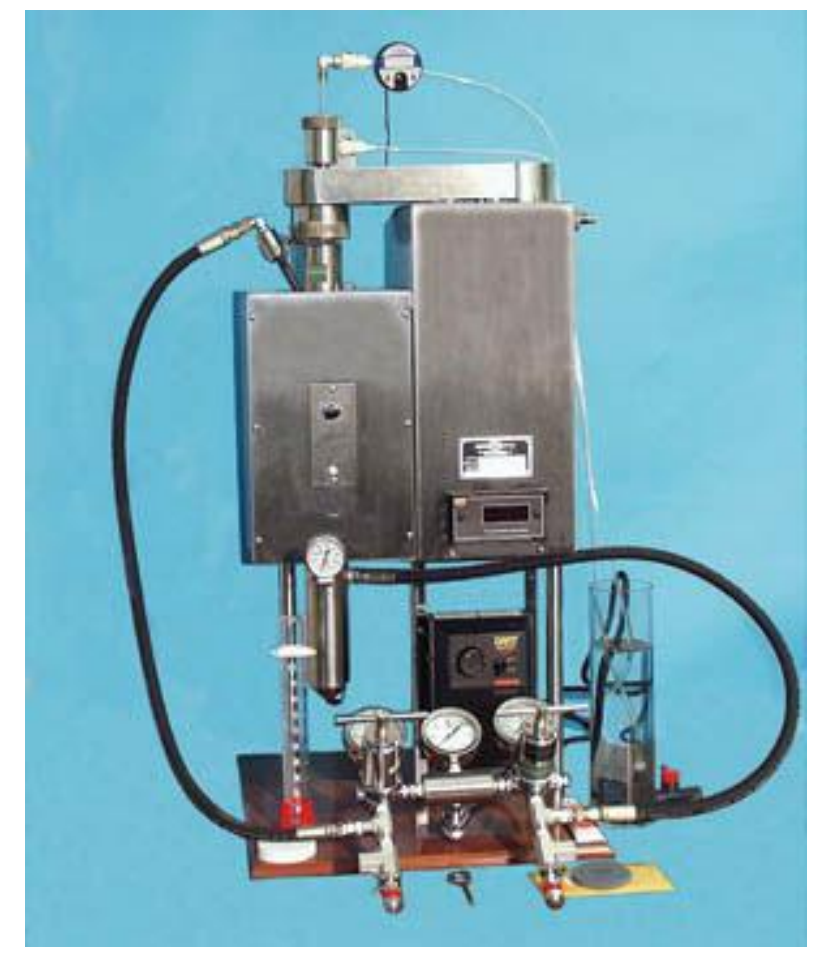

Figura 1- Ofite Dynamic High-Pressure - HTHP.

O filtro mede propriedades de filtração sob diferentes condições dinâmicas de fundo de poço. Equipado com um motor de hélices, que fornece diferentes velocidades no interior de uma célula de $500 \mathrm{~mL}$, podendo conferir fluxo laminar ou turbulento. A energia é conduzida ao motor, por uma correia dentada que é facilmente acessível para o um ajuste rápido ou para a remoção.

Os testes são realizados basicamente em uma cela de filtração, e um meio poroso de cerâmica (FANN), com $6 \mathrm{~cm}$ de diâmetro e $0,63 \mathrm{~cm}$ de espessura. Para a célula são transferidos $400 \mathrm{ml}$ de água destilada e o agente obturante, carbonato de cálcio, a 5\% de 
concentração, possuindo uma densidade de $2885 \mathrm{~kg} / \mathrm{m}^{3}$, está foi medida no picnômetro de hélio.

Após a efetuação dos ensaios no filtro, a torta é pesada e levada para estufa na temperatura de $70^{\circ} \mathrm{C}$ por 48 horas.

Os testes foram feitos nas seguintes pressões em triplicata: 100, 200, 400, $600 \mathrm{e}$ $800 \mathrm{KPa}$, e podem ser representados das seguintes formas:

Tabela 1- Índice de reconhecimento dos testes.

\begin{tabular}{c|c}
\hline \multirow{3}{*}{$\mathrm{P}=100 \mathrm{KPa}$} & Teste 1 \\
& Teste 2 \\
Teste 3 \\
\hline \multirow{3}{*}{$\mathrm{P}=200 \mathrm{KPa}$} & Teste 1 \\
& Teste 2 \\
Teste 3 \\
\hline \multirow{2}{*}{$\mathrm{P}=400 \mathrm{KPa}$} & Teste 1 \\
& Teste 2 \\
& Teste 3 \\
\hline $\mathrm{P}=600 \mathrm{KPa}$ & Teste 1 \\
& Teste 2 \\
Teste 3 \\
\hline \multirow{2}{*}{$\mathrm{P}=800 \mathrm{KPa}$} & Teste 1 \\
& Teste 2 \\
& Teste 3 \\
\hline
\end{tabular}

\subsection{MATERIAIS E MÉTODOS}

Uma massa de carbonato de cálcio é previamente pesada $(61,01 \mathrm{~g})$ e transferida para um recipiente com $400 \mathrm{ml}$ de água destilada. Logo após a mistura é colocada no filtro e agitada por 3 minutos. E a partir disso é medido o tempo a cada $20 \mathrm{ml}$ de volume de filtrado retirados, para obter a permeabilidade.

A torta molhada é retirada, sua massa é aferida e depois colocada na estufa por quarenta e oito horas na temperatura de $80^{\circ} \mathrm{C}$. Após o tempo determinado na estufa, a massa torta é aferida novamente para obter o cálculo da porosidade.

A metodologia proposta por Massarani (2002), para a determinação da concentração de sólidos, resistividade média da torta, resistência do meio filtrante, porosidade, tempo de filtração e volume de filtrado pode ser estabelecida partindo das seguintes considerações e hipóteses simplificadoras:

a) Admitir que a velocidade superficial do sólido na torta seja substancialmente menor do que aquela do líquido;

b) Torta é moderadamente compressível;

Adotando as considerações acima: 


$$
q_{F}=q_{F}(t)
$$

A equação do Movimento para o fluido na torta toma a seguinte forma:

$$
\frac{d}{d z} p(z, t)=-\frac{d}{d z} p_{s}(z, t)=\frac{\mu_{F}}{k} q_{F}(t)
$$

Correlacionando os resultados da filtração com as condições operacionais expressas pela queda de pressão no filtro. Seja M a massa de sólido seco que compõe a torta:

$$
d M=\rho_{s}(1-\varepsilon) A d z
$$

Onde A é área da superfície de filtração. Vem da equação (1):

$$
-d p_{s}=\frac{1}{p_{s}(1-s) k} \times \frac{\mu q_{F}}{A} d M=\alpha(\varepsilon) \frac{\mu q_{F}}{A} d M_{y}
$$

Sendo a resistividade local (L/M),

$$
\alpha=\frac{1}{\rho_{s}(1-s) k(s)}
$$

A torta e o meio filtrante são meios porosos percolados em série pelo fluido. A expressão para a queda de pressão no filtro pode ser estabelecida:

$$
p(l, t)-p\left(-l_{m}, t\right)=\Delta p=\left[<\alpha>\frac{M}{A}+R_{m}\right] \mu_{F} q_{F}
$$

A velocidade superficial do fluido e a massa de sólido seca na torta estão relacionados ao volume de filtração $\mathrm{V}$, ao tempo $\mathrm{t}$, à área de filtração $\mathrm{A}$ e à concentração de sólidos na suspensão, c:

$$
\begin{aligned}
& q_{F}=\frac{1}{A} \frac{d V}{d t} \\
& c \cong \frac{M}{\rho_{F} V}
\end{aligned}
$$

A equação da filtração resulta da combinação das equações (6) a (8),

$$
\frac{d t}{d V}=\frac{\mu_{F}}{A(\Delta p)}\left[\frac{\left\langle\alpha>V \rho_{F^{c}}\right.}{A}+R_{m}\right],<\alpha>=f(\Delta p)
$$

Na maioria das situações de interesse industrial a filtração é conduzida sob queda de pressão constante:

$$
\frac{t}{V}=\frac{\mu_{F}}{A(\Delta p)}\left[\frac{\left\langle\alpha>V \rho_{F} c\right.}{2 A}+R_{m}\right]
$$

\subsection{ANÁLISE DOS RESULTADOS E DISCUSSÕES}

Os dados obtidos para a construção dos perfis de Permeabilidade e de Concentração Volumétrica de Sólidos encontram-se abaixo: 
Tabela 2- Valores de $\alpha$

\begin{tabular}{l|l|l|l|l|l}
\hline \multicolumn{5}{l}{ Valores de $\alpha(\mathrm{g} / \mathrm{cm})$} \\
\hline Pressões & $100 \mathrm{KPa}$ & $200 \mathrm{KPa}$ & $400 \mathrm{KPa}$ & $600 \mathrm{KPa}$ & $800 \mathrm{KPa}$ \\
\hline$\alpha($ teste1) & $4,912 \times 10^{9}$ & $1,402 \times 10^{10}$ & $3,435 \times 10^{10}$ & $5,750 \times 10^{10}$ & $8,059 \times 10^{10}$ \\
$\alpha($ teste2) & $4,810 \times 10^{9}$ & $1,391 \times 10^{10}$ & $3,477 \times 10^{10}$ & $5,570 \times 10^{10}$ & $8,062 \times 10^{10}$ \\
$\alpha($ teste3) & $4,746 \times 10^{9}$ & $1,363 \times 10^{10}$ & $3,446 \times 10^{10}$ & $5,562 \times 10^{10}$ & $8,049 \times 10^{10}$ \\
\hline Média $\alpha$ & $4,823 \times 10^{9}$ & $1,385 \times 10^{10}$ & $3,453 \times 10^{10}$ & $5,627 \times 10^{10}$ & $8,057 \times 10^{10}$ \\
\hline
\end{tabular}

Os dados acima da Tabela 2 foram obtidos pela regressão linear da equação 10 , com os cálculos dos coeficientes angulares, formadas pelos pontos experimentais gerados em cada teste. Conforme o esperado os valores de $\alpha$ aumenta com a pressão.

Tabela 3- Valores de $\varepsilon_{\mathrm{S}}$

Valores de Concentração Volumétrica de Sólidos es

\begin{tabular}{l|l|l|l|l|l}
\hline Ps & $59,3 \mathrm{KPa}$ & $121,4 \mathrm{KPa}$ & $244,8 \mathrm{KPa}$ & $370,8 \mathrm{KPa}$ & $496,8 \mathrm{KPa}$ \\
\hline es (teste 1) & 0,600 & 0,606 & 0,612 & 0,616 & 0,622 \\
ss (teste 2) & 0,601 & 0,609 & 0,612 & 0,619 & 0,622 \\
ss (teste 3) & 0,597 & 0,607 & 0,612 & 0,620 & 0,620 \\
\hline Média & 0,593 & 0,607 & 0,612 & 0,618 & 0,621 \\
\hline
\end{tabular}

Usando os dados da Tabela 3 foi deduzido uma equação constitutiva, equação 11, que modela o comportamento da pressão no sólido (Ps) em função da concentração

volumétrica de sólidos $\left(\varepsilon_{\mathrm{S}}\right)$, com a ajuda do software Statistica 7.0, com um $\mathrm{R}^{2}=0.9993$.

$$
P_{s}=3,653 \times 10^{8} \varepsilon s^{32,7}-155,39
$$

A Tabela 3 possui os dados de concentração volumétrica de sólidos, que com tais resultados e a densidade do sólido obtido com o picnômetro de gás hélio, $2,885 \mathrm{~g} / \mathrm{cm}^{3}$, podemos calcular a permeabilidade (Ks) a partir da equação 5 , os valores são listados na Tabela 4.

Com os dados da Tabela 4 foi chegou-se a equação constitutiva, equação 12, que modela o comportamento da pressão no sólido (Ps) em função da permeabilidade $\left(K_{\mathrm{S}}\right)$, com a ajuda do software Statistica 7.0, com um $R^{2}=0.9995$.

$$
P=3,69 \times 10^{-8} K^{-0,7982}
$$


Tabela 4- Valores de Ks

Valores de Permeabilidade Ks $\left(\mathrm{m}^{2}\right)$

\begin{tabular}{l|l|l|l|l|l}
\hline Pressões & $100 \mathrm{KPa}$ & $200 \mathrm{KPa}$ & $400 \mathrm{KPa}$ & $600 \mathrm{KPa}$ & $800 \mathrm{KPa}$ \\
\hline $\mathrm{K}$ & $1,795 \times 10^{-12}$ & $6,372 \times 10^{-13}$ & $2,587 \times 10^{-13}$ & $1,615 \times 10^{-13}$ & $1,136 \times 10^{-13}$ \\
\hline
\end{tabular}

O perfil de Pressão versus Concentração Volumétrica de Sólidos comportou-se como esperado, a pressão varia como uma função exponencial da concentração volumétrica de sólidos. A concentração de sólidos mostrou-se pouco sensível em relação a variação de pressão na faixa estudada, que foi entre 100 a $800 \mathrm{KPa}$.

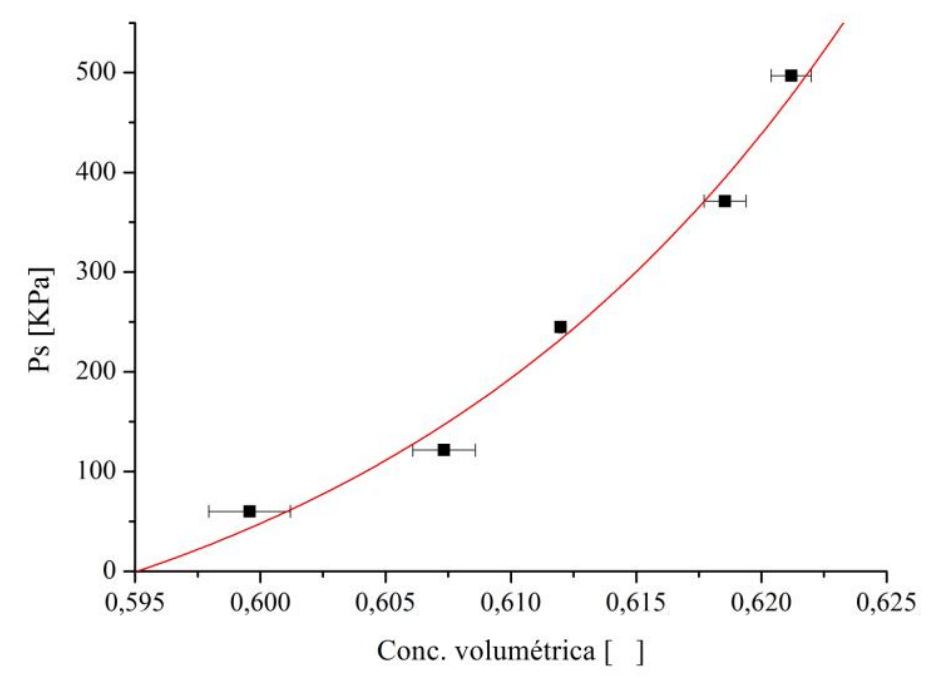

Figura 2- Perfil de Pressão versus Concentração Volumetrica de Sólidos.

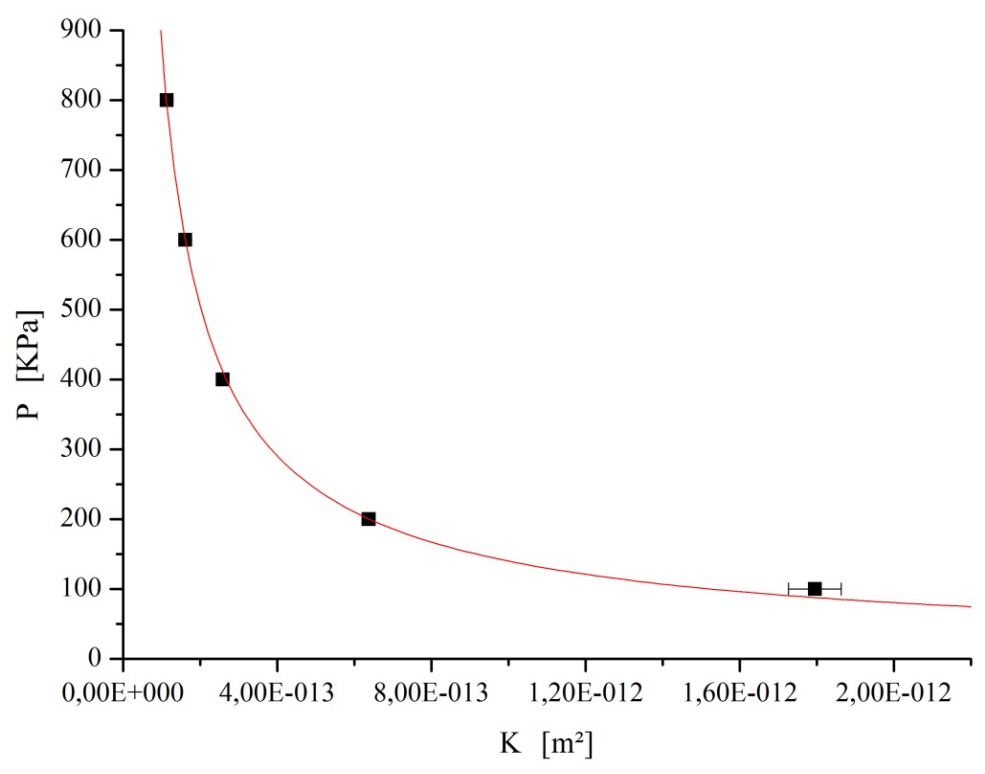

Figura 3- Distribuição de Pressão versus Permeabilidade. 


\subsection{CONCLUSÃO}

A técnica utilizada com o filtro HPHT é uma alternativa bastante viável por ser uma técnica que responde a dados replicados precisamente. Com os dados pode-se obter equações constitutivas para pressão no sólidos e permeabilidade com ajustes satisfatórios que podem ser utilizados para modelar processos de separação sólidolíquido.

Pode-se concluir também que a concentração de sólidos tem pouca variação com a pressão, contrária á permeabilidade que varia expressivamente com a pressão para o carbonato de cálcio utilizado na faixa de pressão de 100 a $800 \mathrm{KPa}$.

\subsection{AGRADECIMENTOS}

“Agradecemos a Fundação de Amparo à Pesquisa do Estado de Minas Gerais (FAPEMIG) pelos recursos concedidos no Projeto de Participação Coletiva em Eventos Técnicos-Científicos (PCE-00082-14)”.

\subsection{REFERÊNCIAS}

MASSARANI, G. Fluidodinâmica em Sistemas Particulados. Rio de Janeiro: E-Papers Serviços Editoriais, 2002.

FERREIRA, A.S; MASSARANI, G.; Physico-mathematical modeling of crossflow filtration, Chemical Engineering Journal, 111, 199-204, 2005.

BOURGOYNE, A.T., MILLHEIM, K.K., CHENEVERT, M.E., YOUNG JR, F.S., Applied drilling engineering, Society of Petroleum Engineers, Richardson, TX, USA, Second Print, citado por: Scheid, C.M.; Martins, A.L.; Massarani, G.; "Escoamento axial de fluidonão-Newtoniano em duto com paredes permeáveis”, 1991.

ARAÚJO, C.A.O; Estudo da Filtração em Geometria Cilíndrica. Tese de Mestrado, UFRRJ, 2010. 